\title{
It is premature to test older drivers with the SIMARD-MD
}

Michel Bédard ${ }^{1,2,3^{*}}$

Shawn Marshall ${ }^{4,5}$

Malcolm Man-Son-Hing ${ }^{4,5}$

Bruce Weaver ${ }^{1,3}$

Isabelle Gélinas ${ }^{6,7}$

Nicol Korner-Bitensky ${ }^{6,7}$

Barbara Mazer ${ }^{6,7}$

Gary Naglie $8,9,10$

Michelle M. Porter ${ }^{11}$

Mark J. Rapoport ${ }^{12}$

Holly Tuokko ${ }^{13}$

Brenda Vrkljan ${ }^{14}$

For the Candrive Research Team

${ }^{1}$ Centre for Research on Safe Driving, Lakehead University, Thunder Bay, Ontario

${ }^{2}$ St. Joseph's Care Group, Thunder Bay, Ontario

${ }^{3}$ Northern Ontario School of Medicine, Thunder Bay, Ontario

${ }^{4}$ University of Ottawa, Ottawa, Ontario

${ }^{5}$ Ottawa Hospital Research Institute

${ }^{6}$ McGill University, Montréal, Québec

${ }^{7}$ Centre for Interdisciplinary Research in Rehabilitation of Greater Montréal, Québec

${ }^{8}$ Department of Medicine and Rotman Research Institute, Baycrest Geriatric Health Care Centre, Toronto, Ontario

${ }^{9}$ Research Department, Toronto Rehabilitation Institute, University Health Network, Toronto, Ontario

${ }^{10}$ Department of Medicine and Institute of Health Policy, Management and Evaluation, University of Toronto, Toronto, Ontario ${ }^{11}$ University of Manitoba

${ }^{12}$ University of Toronto

${ }^{13}$ University of Victoria

${ }^{14}$ McMaster University

*Corresponding author (mbedard@lakeheadu.ca) 


\begin{abstract}
Background: A new tool, the SIMARD-MD, has been proposed to help physicians identify cognitively impaired drivers who may be unfit to drive, but little empirical evidence is available to justify its use. We analyzed data from a cohort of older Canadian drivers who had undergone cognitive testing to: 1) correlate the SIMARD-MD with other tools that measure cognition (e.g., Trail-Making Test), 2) identify how many drivers, using published cut-offs on the SIMARD-MD, would be recommended to lose their license, or be considered fit to drive, or be required to undergo further driving assessment, and 3) determine if the SIMARD-MD is biased by level of education as many cognitive tools are.

Methods: Cross-sectional data from 841 drivers aged 70 and over from seven Canadian sites who are enrolled in a 5-year cohort study were used for the analyses. Scores on the SIMARDMD were correlated with scores on the other cognitive measures. The recommendations that would be made based on the SIMARD-MD scores were based on published cut-off values suggested by the authors of the tool. The impact of education status was examined using linear regression controlling for age.
\end{abstract}

Results: Correlations between the SIMARD-MD and other cognitive measures ranged from .15 to .86 . Using published cut-off scores, 21 participants (2.5\%) would have been recommended to relinquish their licenses, 428 (50.9\%) would have been deemed fit to drive, and 392 (46.6\%) would have been required to undergo further testing. We found a difference of 8.19 points $(95 \% \mathrm{Cl}=4.99,11.40, \mathrm{p}<.001)$ in favour of drivers with post-secondary education versus those without, representing over $11 \%$ of the mean score. 
Discussion: The SIMARD-MD is unlikely to be valuable to clinicians because it lacks sufficient precision to provide clear recommendations about fitness-to-drive. Recommendations based solely on the SIMARD-MD may place many seniors at risk of losing their transportation mobility or incurring unnecessary stress and costs to prove they are safe to drive. Furthermore, the education bias may create an unwanted structural inequity. Hence, adoption of the SIMARD$\mathrm{MD}$ as a tool to determine fitness-to-drive appears premature. 


\section{Introduction}

A new "screening" tool ${ }^{a}$ to identify cognitively impaired drivers who may be unfit to drive was unveiled in the "2010 BC Guide in Determining Fitness to Drive" published by the Office of the Superintendent of Motor Vehicles (OSMV) of British Columbia (BC). The tool, called the "Screening Tool for the Identification of Cognitively Impaired Medically At-Risk Drivers: A modification of the DemTect" (SIMARD-MD) (Dobbs \& Schopflocher, 2010), is a tool that can be administered in a physician's office. As far as we know, BC is the only province to promote its use and it was not specified why this tool was chosen over other tools currently used by health professionals.

The SIMARD-MD is a shorter derivative of the DemTect (Kalbe et al., 2004). The DemTect is a paper and pencil tool that takes less than 10 minutes to complete and that was developed to identify mild cognitive impairment in older adults. It is highly sensitive in detecting mild Alzheimer disease and cognitive impairment and has been proposed by its authors as the first step in diagnosing the presence of cognitive impairment. The DemTect was not, however, developed nor validated to identify unfit drivers.

There is no disputing that drivers who are unsafe because of medical conditions should be identified, ${ }^{\mathrm{c}}$ but this identification is challenging, and often contentious given the negative consequences associated with driving cessation (Mullen \& Bédard, 2009). In Canada, driver licensing is a provincial jurisdiction, and as such there are no uniform testing and reporting requirements. Nonetheless, most Canadian jurisdictions have mandated physicians to report

\footnotetext{
a A "triage" tool may be a better description.

${ }^{\mathrm{c}}$ The maintenance of mobility is crucial. Drivers who are unsafe should be helped to remediate the situation if possible or supported as they make the transition to "non-driving" status.
} 
medically unfit drivers. This is a cause for concern as physicians feel they lack the necessary tools and knowledge to evaluate fitness-to-drive within a clinic setting (Jang et al., 2007). This knowledge gap outlines the need to develop valid and reliable driving assessment tools. However, adoption of these tools should be supported by evidence of adequate benefits and minimal risks (Kitson \& Straus, 2010). Careful appraisal of the only published study supporting the use of the SIMARD-MD raises concerns about methodological limitations and a potential for harm if it were to be applied as a driver screening tool on a large-scale basis (Bédard, Weaver, Man-Son-Hing, Classen, \& Porter, 2011; Hogan \& Bédard, 2011).

As now implemented in BC, the SIMARD-MD uses two cut-off values. Drivers who score $\leq 30$ are flagged as unfit to drive, barring mitigating factors such as a language barrier, and results on this tool alone may be sufficient to result in revocation of the driving privilege. Drivers who score $>70$ are flagged as fit to drive in the absence of mitigating factors such as very high baseline intellect, which can decrease the sensitivity of the tool. Those with scores that fall in between are considered "indeterminate" and are required to undergo a functional assessment to establish their fitness-to-drive. However, in the sole published study on the validity of the SIMARD-MD, $20 \%$ of drivers who scored below the $\leq 30$ cut-off value passed an on-road examination and $13 \%$ of those scoring $>70$ and thus labelled fit-to-drive, failed the onroad examination (Dobbs et al., 2010). Surely, the harm that may come to these individuals (losing the driving privilege when fit to drive or being allowed on the road when unfit to drive), and society at-large, is substantial. Moreover, the remaining $50 \%$ of the individuals tested, who were classified as indeterminate, would require further specialized driving assessment such as 
the protocol offered by the DriveABLE ${ }^{T M}$ company ${ }^{d}$. This step results in a costly assessment, where the cost is often assumed by the individual driver. The tool properties of the SIMARD$M D$ raise questions about the relative benefits versus the potential risks of adopting it as a screening tool for fitness-to-drive.

An additional methodological concern is that cognitive tools are notorious for having an education bias (Crane, van Belle, \& Larson, 2004; Teresi et al., 1995; Tombaugh, Kozak, \& Rees, 1999). The authors of the DemTect recommended adjustment of the score for people with 11 years of education or less. We found no such recommendation in the SIMARD-MD publication (Dobbs et al., 2010).

The purpose of our study was to use data from a cohort of relatively healthy older Canadian drivers to determine: 1 ) if the SIMARD-MD scores correlate with scores from other cognitive tools, 2) how many of these older drivers, based on the SIMARD-MD recommended cut-off scores, would be considered unfit-to-drive versus how many would be considered fit-todrive, or be required to undergo further driving evaluation, and 3) if the SIMARD-MD has an education bias.

\section{Methods}

\section{Participants/procedure}

Starting in 2009, drivers aged 70 and older from seven Canadian cities (Montréal, Ottawa, Toronto, Hamilton, Thunder Bay, Winnipeg, and Victoria) were enrolled in the Candrive Cohort Study (see Marshall et al., - this issue) and are evaluated annually with a number of

\footnotetext{
${ }^{d}$ In the process adopted in $\mathrm{BC}$, this additional testing would be conducted using the DriveAble protocol. DriveAble is a for-profit company that provides both cognitive testing and on-road driving assessments.
} 
tools, including some that measure cognition (see below). During year 2 of the study, we added the DemTect/SIMARD-MD to the assessment protocol. At the time this document was written, 841 participants had completed the DemTect/SIMARD-MD; we used these data for the analyses presented below. For 244 of these participants we also had complete results for other cognitive tools. All scores used for the analyses were obtained at the same annual assessment.

\section{Tools}

The cognitive toos included in the Candrive Study were originally selected because they held potential to identify unfit drivers. After publication of the article on the SIMARD-MD, we added the DemTect to the annual assessment and calculated scores for the SIMARD-MD as described below.

DemTect/SIMARD-MD: The German Demenz-Detektion (DemTect) was developed to identify cognitive impairment in older adults and is sufficiently sensitive to identify mild cognitive impairment and people with early-stage dementia (Kalbe et al., 2004). The tool includes five sub-tests: a word list to-be-recalled, a number trans-coding task, semantic verbal fluency tasks, reverse digit span, and delayed recall of the word list. Possible scores range from 0 to 18 , with one point added to the score in the absence of post-secondary education; higher scores reflect better cognition. The SIMARD-MD relies on three of these sub-tests (number trans-coding, semantic verbal fluency, and delayed recall) and a modified scoring algorithm resulting in scores ranging from 0 to 130 where higher represents better cognition (Dobbs \& Schopflocher, 2010). 
Mini-Mental State Examination: The Mini-Mental State Examination (MMSE)(Folstein, Folstein, \& McHugh, 1975) was also used to assess participants' cognitive function. Possible scores range from 0 to 30 , where 0 indicates severe cognitive impairment and 30 indicates no impairment.

Montreal Cognitive Assessment: The Montreal Cognitive Assessment (MoCAC) is used as a screening tool for mild cognitive impairment (MCl). It has demonstrated excellent sensitivity and specificity in detecting MCl (Nasreddine et al., 2005)(Smith, Gildeh, \& Holmes, 2007). While many of the sub-tests embedded in the MoCA may be associated with driving, to our knowledge it has not been tested formally on its own. Scores can range from 0 to 30, with higher values indicating better cognition.

Motor-Free Visual Perception Test (version 3): The Motor-Free Visual Perception Test (MVPT) (Mercier, Hebert, Colarusso, \& Hammil, 1997) measures various aspects of visualperceptual abilities such as visual analysis, visual discrimination, and figure-ground discrimination. It is associated with on-road driving performance in older drivers referred for driving assessment (Oswanski et al., 2007) as well as older adults with stroke (Mazer, KornerBitensky, \& Sofer, 1998). We used the time required to complete the test in seconds; lower values represent better performance.

Trail-making Test, Parts A and B: The Trail-Making Test is a neuropsychological tool often used to identify organic brain damage (Reitan, 1958). It measures visual-motor tracking and divided attention (Goode et al., 1998). It actually consists of two parts, A and B (TMT A, TMT B). Performance is measured as the number of seconds required to complete the tasks, 
hence, lower values indicate better performance. Higher scores on TMT A (Bédard, Weaver, Darzins, \& Porter, 2008) and TMT B (Classen et al., 2008) are associated with poorer driving skills.

Attention Network Test: The Attention Network Test (ANT), developed by Fan, McCandliss, Sommer, Raz, and Posner (Fan, McCandliss, Sommer, Raz, \& Posner, 2002), is a computerized reaction time test providing measures of alerting, orienting, and conflict efficiency. The ANT program used in the current study is a revised version of the original ANT and is publically available (http://crsd.lakeheadu.ca/crsd-ant/). We used overall reaction times

as the main measure. Weaver et al (Weaver, Bédard, McAuliffe, \& Parkkari, 2009) demonstrated that older drivers' overall response times to the ANT were predictive of simulated driving test results $\left(R^{2}=.564\right)$. Shorter reaction times are indicative of better performance.

\section{Analytical plan}

We present descriptive statistics as well as Pearson correlation coefficients to examine the associations between SIMARD-MD scores and scores obtained with the other tools. SIMARD-MD scores were categorized based on published cut-off values. That is, scores $\leq 30$ indicated drivers who are categorized as unfit, scores $>70$ indicated drivers who are categorized as fit, and scores of 31-70 indicated drivers categorized as indeterminate who would require further driving evaluation to establish fitness to drive). To examine the potential for education bias in the SIMARD-MD and other cognitive tools, we computed linear regressions. The dependent variable was the score on the tool (e.g., SIMARD-MD) and the 
predictor variables were age and education. We centered age on 70 (the minimum age for Candrive) and then divide by 5 so that a one unit increase in age corresponds to a 5-year increase in age. We dichotomized education level as "post-secondary education" - yes/no, as per the DemTect protocol.

\section{Results}

The mean age of the sample was $78.07(S D=4.80)$; approximately $63 \%$ were men and $57 \%$ had more than high-school education. Scores on the SIMARD-MD were approximately normally distributed with a mean of 71.05 (SD $=22.77$, range $=15$ to 130$)$. Descriptive statistics for the other tools reported here are presented in Table 1, as are their correlations with the SIMARD-MD.

The correlation between the SIMARD-MD and DemTect based on the full sample $(\mathrm{n}=841)$ was $.85(95 \% \mathrm{Cl}, .83$ to $.87 ; \mathrm{p}<.001)$; it was $.86(95 \% \mathrm{Cl}, .83, .89, \mathrm{p}<.001)$ when calculated from the sample with complete data for the other tools $(n=244)$. As shown in Table 1, the SIMARD-MD was correlated with other global measures of cognitive impairment (MMSE, MoCA). The SIMARD-MD was also correlated with the remaining tools, albeit to a lesser extent given that they measure more specific constructs.

Using the cut-off values on the SIMARD-MD identified by Dobbs and Schlopfocher and published by the OSMV of BC, 21 participants (2.5\%) would have been deemed unfit-to-drive (score $\leq 30), 428$ (50.9\%) would have been deemed fit-to-drive (score > 70), and the remaining 392 participants (46.6\%) would have required further testing (score from 31 to 70 ). 
Our linear regression model indicated that both age and education were associated with SIMARD-MD scores $(F[2,712)=51.68, p<.001)$. Scores were lower for older participants; for every 5 -year increase in age, scores fell by 7.15 points $(95 \% \mathrm{Cl}=-8.81,-5.50, \mathrm{p}<.001)$. For education, we found a difference of 8.19 points in favor of drivers with post-secondary education $(95 \% \mathrm{Cl}=4.99,11.40, \mathrm{p}<.001)$ - this difference represented over $11 \%$ of the mean score. Similar regression models were run for other cognitive tools (results presented in Table 2) and identified the SIMARD-MD and TMT as particularly vulnerable to age and education level.

\section{Discussion}

As expected, SIMARD-MD scores correlated very strongly with scores on the longer DemTect from which it was derived. However, the DemTect was developed to identify cognitive impairment, not unfit drivers, and research suggests that being flagged with mild cognitive impairment does not necessarily mean one is unfit-to-drive (Witharr, Brouwer, \& Van Zomeren, 2000). Hence, any tool aimed primarily at the identification of cognitive impairment will likely result in misclassification when used to detect unfit drivers, and, importantly, is unlikely to be accepted by patients because of poor face validity.

Using the SIMARD-MD as outlined in the BC guidelines would have required that roughly half of our sample of healthy drivers, who had scores in the indeterminate category, go for further driving assessment. This proportion is similar to that of the validation sample used by Dobbs and Schopflocher. Furthermore, they reported that $20 \%$ of drivers identified as unfit by the SIMARD-MD actually passed the on-road assessment. At the other end of the spectrum, 
$13 \%$ of those identified as fit failed an on-road evaluation. This illustrates the risk for misclassification using their own data.

A determination that requires a driver to give up driving may seriously affect almost every aspect of the person's life including health, well-being, and social and functional abilities (Mullen \& Bédard, 2009). Hence, it must be made using an approach that has proven validity. The validity of a tool rests with its ability to identify, with reasonable accuracy, whether someone has an underlying condition or not. A tool that classifies half of a population as indeterminate and has the potential to misclassify many safe drivers as unfit is not likely to meet the needs of clinicians and policy-makers.

Moreover, the SIMARD-MD appears to have an education bias. Others, including the developers of the DemTect, have suggested that education must be considered when using tools to identify cognitive impairment. Our results support this contention by showing that drivers without post-secondary education scored more than eight points below drivers with more education, placing them at greater risk of being labelled as "unfit" or "indeterminate". Thus the application of the tool may be creating an important structural inequity on the basis of education.

A limitation of our study is the use of a convenience and "healthy" sample of older drivers. We had only a small proportion of participants who fell below the lowest cut-off value on the SIMARD-MD compared to the validation sample of the original study. However, the proportion of individuals in the "indeterminate" category was roughly identical to that found in the same study, and raises questions about the value of a tool with such a wide "gray" area. In 
the end, the results of this study suggest that the implementation of the SIMARD-MD was premature.

The problems arising from premature adoption of tools can be avoided by following a sound knowledge translation (KT) approach such as the "knowledge-to-action process" put forward by Graham and colleagues (Graham et al., 2006), and adopted by the Canadian Institutes of Health Research (ClHR). In this framework, KT is defined as "a dynamic and iterative process that includes synthesis, dissemination, exchange and ethically-sound application of knowledge to improve the health of Canadians, provide more effective health services and products and strengthen the health care system." (http://www.cihrirsc.gc.ca/e/39033.html) An essential component of the KT process is the accumulation of independent studies leading to the synthesis of research findings. Not only are independent replications key to the scientific method, but "synthesis" implies the existence of more than one study to review. Furthermore, appraisal and grading of the independent studies are paramount; decisions resting on studies of poor quality, let alone on only one study, are always questionable. Using such a framework helps us realize that not all new knowledge warrants translation, and that knowledge by itself is never sufficient to warrant translation. Rather, it is the whole process of knowledge creation that supports KT (Brouwers, Stacey, \& O'Connor, 2010). Had the Knowledge-to-Action process been used it is questionable whether the SIMARD-MD would have been adopted.

Given the serious consequences associated with revoking a person's driver's license and the costs associated with further testing, it is incumbent upon researchers to ensure that the 
assessment tools used are truly valid. The solution clearly lies with additional research to assist with determinations of fitness-to-drive. The Candrive Cohort Study will provide further data on the relationship between the SIMARD-MD, as well as other cognitive, physical performance, sensory and comorbidity measures, and driving outcomes such as at-fault crashes, traffic violations and driving behaviors. Hopefully, results from the Candrive Cohort Study and from other studies, will bring us closer to identifying fair, valid, and transparent assessment tools to assess fitness-to-drive. 
Acknowledgements: Candrive is a CIHR-funded research team to support safe driving in older adults, one of its major objectives is to develop a tool to aid clinicians in assessing older drivers' fitness-to-drive. Michel Bédard is supported by a Canada Research Chair in Aging and Health (www.chairs.gc.ca). Gary Naglie is supported by the George, Margaret and Gary Hunt Family Chair in Geriatric Medicine, University of Toronto. 
Table 1. Descriptive statistics and correlations between the SIMARD-MD and other cognitive tools $(\mathrm{N}=244)$.

\begin{tabular}{|l|c|c|c|c|c|c|}
\hline \multicolumn{1}{|c|}{ Tool $^{\text {a }}$} & Minimum & Maximum & Mean & $\begin{array}{c}\text { Standard } \\
\text { Deviation }\end{array}$ & $\begin{array}{c}\text { Correlation with } \\
\text { SIMARD-MD } \\
\text { (95\% CI) }\end{array}$ & $\begin{array}{c}\text { p- } \\
\text { value }\end{array}$ \\
\hline SIMARD-MD & 18 & 122 & 71.4 & 22.64 & --- & --- \\
\hline DemTect & 5 & 19 & 14.8 & 2.95 & $.86(.83, .89)$ & $<.001$ \\
\hline MMSE & 22 & 30 & 28.3 & 1.43 & $.44(.34, .54)$ & $<.001$ \\
\hline MoCA & 18 & 30 & 25.9 & 2.48 & $.37(.25, .47)$ & $<.001$ \\
\hline MVPT & 59 & 360 & 120.3 & 44.11 & $-.28(-.39,-.16)$ & $<.001$ \\
\hline TMT-A & 8 & 78 & 38.8 & 11.37 & $-.33(-.44,-.21)$ & $<.001$ \\
\hline TMT-B & 43 & 310 & 96.6 & 42.78 & $-.32(-.43,-.20)$ & $<.001$ \\
\hline ANT & 590 & 1126 & 787.3 & 76.77 & $-.15(-.27,-.03)$ & .017 \\
\hline
\end{tabular}

${ }^{a}$ SIMARD-MD: Screening Tool for the Identification of Cognitively Impaired Medically At-Risk Drivers: A modification of the DemTect; DemTect: German Demenz-Detektion; MMSE: MiniMental State Examination; MoCA: Montreal Cognitive Assessment; MVPT: Motor-Free Visual Perception Test; TMT-A: Trail-Making Test-Part A; TMT-B: Trail-Making Test-Part B, ANT: Attention Network Test. 
Table 2. Effect of age and education on cognitive tools.

\begin{tabular}{|c|c|c|c|c|c|c|c|}
\hline \multirow[t]{2}{*}{ Tool $^{\mathrm{a}}$} & \multirow[t]{2}{*}{ Variable } & \multirow[t]{2}{*}{$B^{b}$} & \multirow[t]{2}{*}{$S E^{c}$} & \multicolumn{2}{|c|}{$\underline{95 \% \mathrm{Cl}}$} & \multirow[t]{2}{*}{ p-value } & \multirow{2}{*}{$\begin{array}{c}\text { Percentage } \\
\text { of Mean Score }\end{array}$} \\
\hline & & & & Lower & Upper & & \\
\hline \multirow{3}{*}{ SIMARD-MD } & Constant & 78.33 & 1.88 & 74.64 & 82.02 & $<.001$ & --- \\
\hline & (Age-70)/5 & -7.15 & 0.84 & -8.81 & -5.50 & $<.001$ & 10.02 \\
\hline & Education & 8.19 & 1.63 & 4.99 & 11.40 & $<.001$ & 11.47 \\
\hline \multirow{3}{*}{ Demtect } & Constant & 15.75 & 0.24 & 15.27 & 16.23 & $<.001$ & --- \\
\hline & (Age-70)/5 & -0.97 & 0.11 & -1.19 & -0.76 & $<.001$ & 6.60 \\
\hline & Education & 1.07 & 0.21 & 0.66 & 1.49 & $<.001$ & 7.25 \\
\hline \multirow{3}{*}{ MMSE } & Constant & 28.50 & 0.13 & 28.24 & 28.76 & $<.001$ & --- \\
\hline & $($ Age-70)/5 & -0.31 & 0.06 & -0.43 & -0.20 & $<.001$ & 1.10 \\
\hline & Education & 0.54 & 0.11 & 0.32 & 0.76 & $<.001$ & 1.90 \\
\hline \multirow{3}{*}{ MoCA } & Constant & 26.59 & 0.22 & 26.16 & 27.02 & $<.001$ & --- \\
\hline & $($ Age- 70$) / 5$ & -0.72 & 0.10 & -0.91 & -0.53 & $<.001$ & 2.79 \\
\hline & Education & 0.74 & 0.19 & 0.37 & 1.12 & $<.001$ & 2.87 \\
\hline \multirow{3}{*}{ MVPT } & Constant & 111.29 & 3.66 & 104.11 & 118.47 & $<.001$ & --- \\
\hline & $($ Age- 70$) / 5$ & 10.02 & 1.64 & 6.79 & 13.25 & $<.001$ & 8.13 \\
\hline & Education & -7.46 & 3.18 & -13.70 & -1.22 & .019 & 6.06 \\
\hline \multirow{3}{*}{ TMT-A } & Constant & 35.28 & 1.20 & 32.92 & 37.63 & $<.001$ & --- \\
\hline & (Age-70)/5 & 3.70 & 0.54 & 2.64 & 4.75 & $<.001$ & 9.33 \\
\hline & Education & -2.91 & 1.04 & -4.95 & -0.87 & .005 & 7.35 \\
\hline \multirow{3}{*}{ TMT-B } & Constant & 82.58 & 3.61 & 75.49 & 89.66 & $<.001$ & --- \\
\hline & $($ Age- 70$) / 5$ & 14.44 & 1.62 & 11.27 & 17.62 & $<.001$ & 14.78 \\
\hline & Education & -14.42 & 3.13 & -20.57 & -8.27 & $<.001$ & 14.76 \\
\hline \multirow{3}{*}{ ANT } & Constant & 779.11 & 11.61 & 756.23 & 801.98 & $<.001$ & -- \\
\hline & $($ Age- 70$) / 5$ & 13.76 & 5.40 & 3.11 & 24.40 & .012 & 1.75 \\
\hline & Education & -19.19 & 9.86 & -38.61 & 0.22 & .053 & 2.43 \\
\hline
\end{tabular}

${ }^{a}$ SIMARD-MD: Screening Tool for the Identification of Cognitively Impaired Medically At-Risk Drivers: A modification of the DemTect; DemTect: German Demenz-Detektion; MMSE: MiniMental State Examination; MoCA: Montreal Cognitive Assessment; MVPT: Motor-Free Visual Perception Test; TMT-A: Trail-Making Test-Part A; TMT-B: Trail-Making Test-Part B, ANT: Attention Network Test. ${ }^{\mathrm{b}}$ Regression coefficient 'Standard Error 


\section{References}

Bédard, M., Weaver, B., Darzins, P., \& Porter, M. M. (2008). Predicting driving performance in older adults: We are not there yet! Traffic Injury Prevention, 9 (4), 336-341.

Bédard, M., Weaver, B., Man-Son-Hing, M., Classen, S., \& Porter, M. M. (2011). The SIMARD screening tool to identify unfit drivers: Are we there now? Journal of Primary Care and Community Health, 2(2), 133-135.

Brouwers, M., Stacey, D., \& O'Connor, A. (2010). Knowledge creation: Synthesis, tools and products. Canadian Medical Association Journal, 182(2), E68-72.

Classen, S., Horgas, A., Awadzi, K., Messinger-Rapport, B., Shechtman, O., \& Joo, Y. (2008). Clinical predictors of older driver performance on a standardized road test. Traffic Injury Prevention, 9(5), 456-462.

Crane, P. K., van Belle, G., \& Larson, E. B. (2004). Test bias in a cognitive test: Differential item functioning in the CASI. Statistics in Medicine, 23(2), 241-256.

Dobbs, B. M., Broeren, T., Craig, S., Gamble, C., Grant, B., McCloskey, M., et al. (2010). 2010 BC guide in determining fitness to drive No. Version 1.4). British Columbia: Ministry of Public Safety and Solicitor General Office of the Superintendent of Motor Vehicles.

Dobbs, B. M., \& Schopflocher, D. (2010). The introduction of a new screening tool for the identification of cognitively impaired medically at-risk drivers: The SIMARD A modification of the DemTect. Journal of Primary Care and Community Health, 1(2), 119-127. 
Fan, J., McCandliss, B. D., Sommer, T., Raz, A., \& Posner, M. I. (2002). Testing the efficiency and independence of attentional networks. Journal of Cognitive Neuroscience, 14(3), 340-347.

Folstein, M., Folstein, S., \& McHugh, P. (1975). Mini-mental state: A practical method for grading the cognitive state of patients for the clinicians. Journal of Psychiatric Research, 12, 189-198.

Goode, K. T., Ball, K. K., Sloane, M., Roenker, D. L., Roth, D. L., Myers, R. S., et al. (1998). Useful field of view and other neurocognitive indicators of crash risk in older adults. Journal of Clinical Psychology in Medical Settings, 5(4), 425-440.

Graham, I. D., Logan, J., Harrison, M. B., Straus, S. E., Tetroe, J., Caswell, W., et al. (2006). Lost in knowledge translation: Time for a map? The Journal of Continuing Education in the Health Professions, 26(1), 13-24.

Hogan, D. B., \& Bédard, M. (2011). Papers that might change your practice: Review of the introduction of a new screening tool for the identification of cognitively impaired medically at-risk drivers. Canadian Geriatrics Journal, 14(2), 51-54.

Jang, R. W., Man-Son-Hing, M., Molnar, F. J., Hogan, D. B., Marshall, S. C., Auger, J., et al. (2007). Family physicians' attitudes and practices regarding assessments of medical fitness to drive in older persons. Journal of General Internal Medicine, 22(4), 531-543.

Kalbe, E., Kessler, J., Calabrese, P., Smith, R., Passmore, A. P., Brand, M., et al. (2004). DemTect: A new, sensitive cognitive screening test to support the diagnosis of mild cognitive 
impairment and early dementia. International Journal of Geriatric Psychiatry, 19(2), 136143.

Kitson, A., \& Straus, S. E. (2010). The knowledge-to-action cycle: Identifying the gaps. Canadian Medical Association Journal, 182(2), E73-7.

Mazer, B. L., Korner-Bitensky, N. A., \& Sofer, S. (1998). Predicting ability to drive after stroke. Archives of Physical Medicine and Rehabilitation, 79(7), 743-750.

Mercier, L., Hebert, R., Colarusso, R. P., \& Hammil, D. D. (1997). In Academic Therapy Publications (Ed.), Motor-free visual perception test - vertical format.

Mullen, N., \& Bédard, M. (2009). The end of driving. In M. Odell (Ed.), Older road users: Myths and realities, A guide for medical and legal professionals (pp. 281-292). Tucson, AZ: Lawyers \& Judges.

Nasreddine, Z. S., Phillips, N. A., Bedirian, V., Charbonneau, S., Whitehead, V., Collin, I., et al. (2005). The montreal cognitive assessment, MoCA: A brief screening tool for mild cognitive impairment. Journal of the American Geriatrics Society, 53(4), 695-699.

Oswanski, M. F., Sharma, O. P., Raj, S. S., Vassar, L. A., Woods, K. L., Sargent, W. M., et al. (2007). Evaluation of two assessment tools in predicting driving ability of senior drivers. American Journal of Physical Medicine and Rehabilitation, 86(3), 190-199. 
Reitan, R. M. (1958). Validity of the trail making test as an indicator of organic brain damage. Perceptual and Motor Skills, 8, 271-276.

Smith, T., Gildeh, N., \& Holmes, C. (2007). The montreal cognitive assessment: Validity and utility in a memory clinic setting. Canadian Journal of Psychiatry, 52(5), 329-332.

Stanbrook, M. B., \& Hebert, P. C. (2010). Access to treatment for multiple sclerosis must be based on science, not hope. Canadian Medical Association Journal, 182(11), 1151.

Teresi, J. A., Golden, R. R., Cross, P., Gurland, B., Kleinman, M., \& Wilder, D. (1995). Item bias in cognitive screening measures: Comparisons of elderly white, afro-american, hispanic and high and low education subgroups. Journal of Clinical Epidemiology, 48(4), 473-483.

Tombaugh, T. N., Kozak, J., \& Rees, L. (1999). Normative data stratified by age and education for two measures of verbal fluency: FAS and animal naming. Archives of Clinical Neuropsychology, 14(2), 167-177.

Weaver, B., Bédard, M., McAuliffe, J., \& Parkkari, M. (2009). Using the attention network test to predict driving test scores. Accident Analysis and Prevention, 41(1), 76-83.

Witharr, F. K., Brouwer, W. H., \& Van Zomeren, A. H. (2000). Fitness to drive in older drivers with cognitive impairment. Journal of the International Neuropsychological Society, 6, 480490. 
Woloshin, S., \& Schwartz, L. M. (2006). What's the rush? the dissemination and adoption of preliminary research results. Journal of the National Cancer Institute, 98(6), 372-373. 